\title{
Pulmonary and Chest Wall Metastasectomy in Urogenital Tumors: A Single Center Experience and Review of Literature
}

\author{
Seyd Hossein Fattahi Masoum ${ }^{1}$; Behzad Feizzdeh Kerigh ${ }^{2, *}$; Alireza Goreifi $^{3}$ \\ ${ }_{1}^{1}$ Transplant Research Center, Imam Reza Hospital, Faculty of Medicine, Mashhad University of Medical Sciences, Mashhad, IR Iran \\ ${ }^{2}$ Minimally Invasive Surgery Research Center, Kidney Transplantation Complications Research Center, Ghaem Medical Hospital, Faculty of Medicine, Mashhad University of Medi- \\ cal Sciences, Mashhad, IR Iran \\ ${ }^{3}$ Department of Urology, Mashhad University of Medical Sciences, Mashhad, IR Iran \\ *Corresponding author: Behzad Feizzdeh Kerigh, Minimally Invasive Surgery Research Center, Kidney Transplantation Complications Research Center, Ghaem Medical Hospital, \\ Faculty of Medicine, Mashhad University of Medical Sciences, Mashhad, IR Iran. Tel:+98-5118012857, Fax:+98-5118417404, E-mail: feizzadehb@mums.ac.ir
}

Received: January 7, 2014; Accepted: March 15, 2014

\begin{abstract}
Background: Pulmonary metastases are often found in advanced malignancies. Urogenital malignancies originating from kidney, prostate, testes, and bladder all metastasize preferentially to the lungs.

Objectives:This retrospective study aimed to evaluate the results of pulmonary and chest wall metastasectomy in patients with primary urogenital Tumors.

Patients and Methods: The patients who underwent pulmonary metastasectomy in Ghaem Hospital from 1996 to 2011 were examined. Thirteen out of 79 patients referred for pulmonary metastasectomy to a single thoracic surgeon had metastases from urogenital tumors; two cases with metastasis from urogenital tumors were inoperable. We reviewed their demographic data and also clinicopathological features. Disease free interval (DFI) was defined as the time between the first curative surgery and the appearance of the signs and symptoms of pulmonary metastasis.

Results: Among 11 patients who underwent surgery consisted of eight males and three females. Their metastasis originated from testis tumors $(n=5)$, renal cell carcinoma (RCC; $n=4$ ), bladder tumor $(n=1)$, and prostate cancer $(n=1)$. Their mean age was 41.27 years (range, 21-67). The mean age of the patients with RCC and testis tumor at the time of diagnosing metastasis was 54 and 24.8 years, respectively. There were two other patients (a 62-year-old female and a 54-year-old male) with pleural effusion due to metastatic RCC whose tumor was inoperable because of their poor general condition and hence, were referred for chemotherapy.

Conclusions: Pulmonary metastasectomy is feasible in selected cases.
\end{abstract}

Keywords:Pulmonary; Thoracic Wall; Urogenital Neoplasm; Neoplasm Metastasis

\section{Background}

Pulmonary metastases are often found in advanced malignancies. Urogenital malignancies originating from kidney, prostate, testicles, and bladder tumors metastasize preferentially to the lungs. The diagnosis of lung metastasis is often associated with a very poor prognosis and a short survival time. Consequently, few patients survive more than one year after diagnosis. In these situations, palliative chemotherapy is usually initiated; however, the possibility of metastasectomy should be considered $(1,2)$. Many studies have been conducted on pulmonary metastasectomy and it has become the standard of therapy for various lungs metastases from solid malignancies. Metastases of the primary tumors that do not respond well to chemotherapy, radiotherapy, or a combination of them are especially well suited for surgical resection. If metastases are restricted to the lungs, the use of surgery along with the overall oncological treatment is justified. In patients with widespread diffuse pulmonary metasta- sis or in those whose lesions are technically or functionally inoperable, local interventions such as surgery and radiotherapy are at best palliative. The standard procedure is a circumscribed atypical (lung tissue sparing) resection; rarely, anatomical resection such as pulmonary segmentectomy or lobectomy is required $(1,3)$.

\section{Objectives}

In this study, we presented our single center experience with pulmonary and chest wall metastasectomy of urogenital cancers and reviewed the studies concerning this issue.

\section{Patients and Methods}

We retrospectively examined patients who underwent pulmonary metastasectomy in Ghaem Hospital from 1996 to 2011. From 79 patients referred for pulmonary me 
Fattahi Masoum SH et al.

\begin{tabular}{lcccr}
\hline \multicolumn{1}{l}{ Table 1. Summary of Patients Data } & & & \\
\hline Case & Age, $\mathbf{y} / \mathrm{Gender}^{\mathrm{a}}$ & DFI & Initial Pathology & Site of Metastasis/Type of Surgery \\
\hline $\mathbf{1}$ & $60 / \mathrm{M}$ & 3 & RCC & Chest wall/resection and reconstruction \\
$\mathbf{2}$ & $56 / \mathrm{F}$ & 3 & RCC & Lt lung/sup lobectomy and wedge resection \\
$\mathbf{3}$ & $45 / \mathrm{F}$ & 6 & RCC & Both lungs/metastasectomy \\
$\mathbf{4}$ & $55 / \mathrm{M}$ & 1 & RCC & chest wall/resection and reconstruction \\
$\mathbf{5}$ & $67 / \mathrm{F}$ & 4 & Bladder TCC & Lt lung (sup lobe)/lobectomy \\
$\mathbf{6}$ & $47 / \mathrm{M}$ & 4 & Prostate sarcoma & Lt lung (sup lobe)/lobectomy \\
$\mathbf{7}$ & $26 / \mathrm{M}$ & 6 & Seminoma & Rt lung/Rt lung pneumonectomy \\
$\mathbf{8}$ & $32 / \mathrm{M}$ & 4 & Mixed GCT of testis & Lt lung/wedge resection and segmentectomy \\
$\mathbf{9}$ & $22 / \mathrm{M}$ & 5 & Mixed GCT of testis & Rt lung/metastasectomy \\
$\mathbf{1 0}$ & $23 / \mathrm{M}$ & 3 & Mixed GCT of testis & Rt lung/Inf and Mid segmentectomy \\
$\mathbf{1 1}$ & $21 / \mathrm{M}$ & 2 & Mixed GCT of testis & Rt lung/metastasectomy \\
\hline
\end{tabular}

${ }^{\mathrm{a}}$ Abbreviation: M, male; F, female; RCC, renal cell carcinoma; TCC, transitional cell carcinoma (urothelial carcinoma); DFI, disease free interval; GCT, germ cell tumor; Rt, right; Lt, left; Sup, superior; Inf, inferior; and Mid, middle.

tastasectomy to a single thoracic surgeon, there were 13 cases of urogenital metastases of which two cases were inoperable. We reviewed their demographic data as well as clinicopathological features. Disease free interval (DFI) was defined as the time between the first curative surgery and the appearance of signs and symptoms of pulmonary metastasis.

\section{Results}

Patients with metastases originating from urogenital cancers who underwent surgery consisted of eight males and three females. Their primary tumor was testis tumor $(n=5)$, RCC $(n=4)$, bladder tumor $(n=1)$, and prostate cancer $(n=1)$. Their mean age was 41.27 years (range, 21-67). The mean age of patients with RCC and testis tumor at the time of diagnosing the metastasis was 54 and 24.8 years, respectively. There were two other patients (A 62 -year-old female and a 54-year-old male) with pleural effusion due to metastatic RCC who were found inoperable due to their poor general condition and were referred for chemotherapy.

Clinical presentation of the metastasis was dyspnea and cough in five patients and hemoptysis in one patient. Five patients were asymptomatic and their metastasis was diagnosed in their routine follow-up. Mean DFI was 3.73 years (range, 1-6) in all patients and 2.75 and four years in patients with RCC and testis tumor, respectively.

The site of metastasis was chest wall in two, right lung in four, and left lung in four patients. One patient had bilateral lung metastases. All surgeries were done by a single thoracic surgeon. Two patients with chest wall involvement underwent tumor resection. Chest wall was reconstructed using latissimus dorsi muscle and Mersilene mesh. The other types of surgical procedures included lobectomy (three cases), metastasectomy (three cases), segmentectomy (two cases), and pneumonectomy (one patient; Table 1).

No major complication occurred during surgery and in postoperation period. All patients were discharged with good condition. One patient (case 6 in Table 1) had hemophilia that was prepared for surgery with 100 IU of factor VIII and did not experience any complication in perioperative period.

\section{Discussion}

Systemic metastases occur in about $20 \%$ of testicular germ cell tumors (GCTs), 25\% to 30\% of prostate cancers, $30 \%$ of urothelial carcinoma with muscle invasion, and $50 \%$ of RCCs; in addition, all of them metastasize preferentially to the lungs (4). We reviewed the studies concerning pulmonary metastasectomy in urogenital tumors as well as presenting our experience in this issue.

\subsection{Renal Cell Carcinoma}

RCC metastasizes preferentially to the lung either hematogenous or via lymphatic route (1). At the time of diagnosis, about $20 \%$ of patients show regional or systemic metastases and up to $30 \%$ of them present with metastases after radical nephrectomy (5). Lung and lymph nodes are the most frequent sites of metastasis (62\%-77\% and $34 \%-58 \%$, respectively); the skeletal system and liver are involved less frequently $(18 \%-30 \%)(4,6)$.

Although development of new drugs like tyrosine kinase inhibitors and mTOR (mammalian target of rapamycin) inhibitors is associated with an improvement in progression-free survival, lung metastasectomy remains as an acceptable choice for curative treatment (7). In 21 studies published from 1961 to 2011, the five-year survival after RCC pulmonary metastasectomy ranged from $21 \%$ to $60 \%$ (8). on the other hand, in a study of eight selected patients without extrapulmonary metastases who underwent complete resection of solitary or mul- 
tiple unilateral or bilateral RCC pulmonary metastases, five-year survival was 83\% (9). In most studies, complete resection of metastasis was a good prognostic factor. Although resectability itself may be associated with better prognosis, reduction of tumor burden may also play a significant role. Higher number of metastases and lymph node involvement indicate advanced disease and may compromise complete resectability $(8,10)$. Other good prognostic factors include solitary metastasis (vs. multiple), smaller number of metastases $(<3$ or $<7)$, smaller size of metastases $(<3 \mathrm{~cm})$, metachronous (vs. synchronous) metastases, longer DFI (>12-36 months), absence of positive hilar and/or mediastinal lymph nodes, absence of pleural infiltration, and absence of positive lymph nodes at initial nephrectomy (8). Simultaneous metastasis of thoracic lymph nodes occurs in 30\% to $45 \%$ of cases and is associated with a much less favorable prognosis. Mean survival of these patients has been reported as 26 to 29 months, which is shorter in comparison to 64 to 92 months in patients without lymph node metastases. Therefore, patients with mediastinal lymph node metastases are not suitable for curative surgery (11). Surgery should resect all existing metastases, which may leads to a five-year survival rate of $40 \%$ to $50 \%$ and a mean survival time from 35 to 55 months, with low surgical mortality and morbidity rates (0\%-2\% and 1.5\%-10\%, respectively) (4). Pastorino et al. reported that the five- and ten-year survival rates of patients who underwent complete surgical resection were $36 \%$ and $26 \%$, respectively, which were longer in comparison with the rates in those with incomplete resections (13\% and $7 \%$ in five- and ten-years rates, respectively) (12). Tanguay et al. reported the potential benefits of combining surgery with systemic therapy. Median time to relapse was six months in patients treated with initial surgery and 8.5 months in patients treated with delayed surgery, although the latter group of patients had substantially more sever disease. Among the patients who underwent initial surgery, 55\% had survived after median follow-up of 48 months while $66 \%$ of patients who received initial systemic therapy had survived 27 months. The authors emphasized that the disease burden was much greater in patients who had received initial systemic therapy; however, the promising results seem to support initial systemic therapy for patients with greater disease burden (13). Although DFI, defined as the time interval between nephrectomy and pulmonary metastasectomy or between nephrectomy and diagnosis of pulmonary metastases, varies in metachronous metastases, it does not exceed five years in the majority of cases. However, some others reported a maximum DFI of longer than 15 years $(14,15)$. Very late metastasectomy has been rarely reported, and the long-term results are practically lacking (8).

In our experience, we had four cases of metastatic RCC with mean DFI of 2.75 years, and the mean age of patients at the time of diagnosis of metastasis was 54 years. Two patients had chest wall involvement and two others had lung involvement. Moreover, we had two patients with pleural effusion due to metastatic RCC who were inoperable.

\subsection{TesticularTumors}

The lung is the most common site of metastases in patients with testicular GCTs (16). Pulmonary metastasectomy in GCTs results in five-year survival rate of 65\% (12), the best survival rate in comparison to the rates from metastasectomy of other tumor types including epithelial tumors, sarcomas, and melanomas. The role of pulmonary metastasectomy in the treatment of testicular GCT has been evolving with the introduction of cisplatin to chemotherapy regimens. Before cisplatin era, the fiveyear survival rate for patients after pulmonary metastasectomy was $41 \%$ that increased to $65 \%$ after introducing cisplatin (17).

Numerous studies have demonstrated the prognostic value of the resected residual lesions histology. In their study, Einhorn et al. concluded that surgical resection of residual disease following chemotherapy-induced cytoreduction with platinum combination chemotherapy may be therapeutic in some cases and helps to define the optimal subsequent treatment strategy (18). In nonseminomatous testicular cancer, all lesions that remain after chemotherapy should be removed because in up to $35 \%$ of cases, their histopathological findings are inconsistent with their retroperitoneal findings $(1,4)$. Normalization of the tumor markers after chemotherapy does not imply that removing the residual tumors in the lungs and mediastinum is not needed (1). Patients with elevated serum markers were traditionally believed as unresectable and not suitable for surgery; however, a few studies have demonstrated that salvage surgery may have potential for cure in a selected patients with lesions limited to one site (17). Murphy et al. reported that six patients with disease limited to the lung parenchyma and mediastinum had no evidence of disease with a minimum follow-up period of 31 months (19). Wood et al. reported that metastasectomy was performed for three patients with lung metastases and one with mediastinal disease of which two patients were disease-free at 16-month later followup (20). Moreover, in a study by Liu et al., eight patients had persistently elevated serum tumor markers after chemotherapy prior to pulmonary metastasectomy. After metastasectomy, five patients survived without evidence of disease, three of which were considered as cured with follow-up period of longer than 15 years (17).

In general, indications of resecting lung metastatic foci include residual tumors after chemotherapy and normalization of tumor marker values, lack of response to chemotherapy, partial response to chemotherapy, and recurrence after chemotherapy (1). The histological discordance between the two lungs is only $5 \%$; hence, the decision for or against surgical resection of contralateral metastasis may be made on the basis of histological find- 
Fattahi Masoum SH et al.

ings of the first procedure (4).

In our study we had five cases of testicular tumor, four cases of mixed GCTs and one case of seminoma. All of them had normal tumor markers before metastasectomy and their mean DFI was four years.

\subsection{Urothelial Carcinoma}

Locoregional or systemic recurrence may be seen respectively in $5 \%$ and $35 \%$ of patients with muscle-invasive urothelial carcinoma (transitional cell carcinoma) of the urinary bladder after radical cystectomy $(21,22)$. The standard treatment consists of systemic chemotherapy which leads to partial or complete remission in 70\% of patients with mean progression-free interval of seven months, and shorter than 14 months mean survival time (23). Recurrences occur usually at the site of the primary metastasis, which indicates persistence of active cancerous cells; hence, metastasectomy is reasonable to improve the prognosis (4).

Cowles et al. reported surgery for metastatic urothelial cancer for the first time. They observed a long-term disease control in six patients after the resection of a solitary pulmonary metastasis (24). Siefker-Radtke et al. reported outcomes of 31 patients undergoing postchemotherapy resection of metastases of which $77 \%$ were lung metastases. Overall survival (OS) since the time of surgery was 23 months and five-year survival rate was 33\% (25). Abe et al. observed a median survival time of 42 months in 12 patients, with visible cancer in $83 \%$ of them, who underwent metastasectomy at multiple sites including the lungs (26). In a recent study by Lehmann et al., outcomes of 44 patients from 15 different centers in Germany were reported. The patients had distant metastases of the urothelial or upper urinary tract tumors and underwent complete resection of all detectable metastases. OS from the time of resection was 27 months and 7 (15.9\%) patients survived for more than two years without disease progression (27). Recently, Siefker-Radtke et al. presented data from a phase II clinical trial of sequential neoadjuvant chemotherapy with ifosfamide, doxorubicin, and gemcitabine followed by cisplatin, gemcitabine, and ifosfamide in locally advanced urothelial carcinoma. In their report, 35 patients underwent surgical consolidation, including 24 with nodal metastasis, six with tumor fixed to the pelvic sidewall, and five with metastasis to other organs such as lung, brain, abdominal wall, or ileum. Fiveyear OS for these patients was 29\%; moreover, the greatest improvement was seen in patients undergoing surgical consolidation after a $90 \%$ or greater response to chemotherapy (28).

The available evidence suggests that a selected group of patients benefit from surgical consolidation of visceral metastases, most frequently in the setting of lung metastasis. Although there is no standard guideline in this setting, Svatek et al. considered surgical consolidation of visceral metastases for patients with tumor at one distant site who had responded well to chemotherapy and had no evidence of rapid progression elsewhere. In addition, before considering surgical consolidation, they observed patients for three to six months following chemotherapy to exclude rapid progression (29).

In our experience, we had only one case of bladder carcinoma that underwent pulmonary lobectomy with DFI of four years.

\subsection{Prostate Carcinoma}

Pulmonary metastases are found in $5 \%$ to $27 \%$ of prostate cancer cases and usually only after bone metastasis (30, 31). These metastases may present in a diffuse interstitial pattern representing lymphatic spread, which is the most common form, or a multinodular pattern representing hematogenous spread, which is seen in $8 \%$ to $20 \%$ of those with positive radiographic findings. Solitary pulmonary nodules have been reported but are extremely rare (32). Recently, modern diagnostic imaging methods such as choline PET/CT (positron emission tomography/computed tomography) have become essential tools by enabling early diagnosis of isolated metastases in patients with low-PSA recurrence, which can raises patients' hopes of cure (33). Salvage metastasectomy is a surgical treatment of locoregional or solitary systemic metastases that are confirmed by imaging studies after local primary or systemic therapy. The aim of salvage metastasectomy in prostate cancer is to gain time before initiating systemic androgen deprivation, which is associated with marked side effects that are time-limited. This treatment is not helpful to increase the survival time. Due to the limited life expectancy, visceral metastasectomy or, alternatively, radiotherapy should be performed only if the patient's symptoms cannot be controlled by conservative management or if his function is threatened (4).

In our experience we had only one case of prostate sarcoma which underwent lobectomy with DFI of four years. The patient has been operated because of his severe respiratory symptoms that could not be controlled by conservative management.

The diagnosis of lung metastases in urogenital tumors is often associated with a very poor prognosis and a short survival time. The primary treatment in these patients is palliative chemotherapy. However, an interdisciplinary tumor board should also discuss the possibility of metastasectomy. The aim of treatment in these situations is palliative local radical resection and symptom control. Survival after lung metastasectomy depends on the nature of the primary tumor. In the case of urogenital tumors, pulmonary metastasectomy can be performed in selected patients and may improve the survival rate as well as quality of life. Therefore, the decision for or against metastasectomy must be made on a case-by-case basis.

\section{Acknowledgements}

We thank Mrs. Yaghouti for her excellent cooperation. 


\section{Author's Contribution}

Both authors worked equally.

\section{Financial Disclosure}

There was no financial interest.

\section{Funding/Support}

There was no funding source.

\section{References}

1. Pfannschmidt J, Egerer G, Bischof M, Thomas M, Dienemann H. Surgical intervention for pulmonary metastases. Dtsch Arzteb Int. 2012;109(40):645-51.

2. Younes RN, Haddad F, Ferreira F, Gross JL. [Surgical removal of pulmonary metastasis: prospective study in 182 patients]. Rev Assoc Med Bras. 1998;44(3):218-25.

3. Kim JJ, Park JK, Wang YP. Surgical resection of pulmonary metastasis from renal cell carcinoma. Korean J Thorac Cardiovasc Surg. 2011;44(2):159-64

4. Heidenreich A, Wilop S, Pinkawa M, Porres D, Pfister D. Surgical resection of urological tumor metastases following medical treatment. Dtsch Arztebl Int. 2012;109(39):631-7.

5. Beisland C, Medby PC, Beisland HO. Presumed radically treated renal cell carcinoma--recurrence of the disease and prognostic factors for subsequent survival. Scand J Urol Nephrol. 2004;38(4):299-305.

6. Mekhail TM, Abou-Jawde RM, Boumerhi G, Malhi S, Wood L, Elson $P$, et al. Validation and extension of the Memorial Sloan-Kettering prognostic factors model for survival in patients with previously untreated metastatic renal cell carcinoma. J Clin Oncol. 2005;23(4):832-41.

7. Staehler M. The role of metastasectomy in metastatic renal cell carcinoma. Nat Rev Urol. 2011;8(4):180-1.

8. Tsakiridis K, Visouli AN, Zarogoulidis P, Mpakas A, Machairiotis $\mathrm{N}$, Stylianaki A, et al. Lost in time pulmonary metastases of renal cell carcinoma: complete surgical resection of metachronous metastases, 18 and 15 years after nephrectomy. J Thorac Dis. 2012;4(Suppl 1):69-73.

9. Chen F, Fujinaga T, Shoji T, Miyahara R, Bando T, Okubo K, et al. Pulmonary resection for metastasis from renal cell carcinoma. Interact Cardiovasc Thorac Surg. 2008;7(5):825-8.

10. Pogrebniak HW, Haas G, Linehan WM, Rosenberg SA, Pass HI. Renal cell carcinoma: Resection of solitary and multiple metastases. The Annals of Thoracic Surgery. 1992;54(1):33-8.

11. Pfannschmidt J, Klode J, Muley T, Dienemann H, Hoffmann H. Nodal involvement at the time of pulmonary metastasectomy: experiences in 245 patients. Ann Thorac Surg. 2006;81(2):448-54.

12. Pastorino U, Buyse M, Friedel G, Ginsberg RJ, Girard P, Goldstraw $\mathrm{P}$, et al. Long-term results of lung metastasectomy: Prognostic analyses based on 5206 cases. J Thoracic Cardiovasc Surg. 1997;113(1):37-49.

13. Tanguay S, Swanson DA, Putnam JBJ. Renal Cell Carcinoma Metastatic to the Lung: Potential Benefit in the Combination of Biological Therapy and Surgery.J Urol.1996;156(5):1586-9.

14. Kanzaki R, Higashiyama M, Fujiwara A, Tokunaga T, Maeda J, Okami J, et al. Long-term results of surgical resection for pulmonary metastasis from renal cell carcinoma: a 25-year singleinstitution experience. Eur J Cardiothorac Surg. 2011;39(2):167-72.

15. Shiono S, Yoshida J, Nishimura M, Nitadori J, Ishii G, Nishiwaki Y, et al. Late pulmonary metastasis of renal cell carcinoma resected 25 years after nephrectomy.Jpn J Clin Oncol. 2004;34(1):46-9.
16. Kawamukai K, Di Saverio S, Antonacci F, Lacava N, Boaron M Mediastinal germ cell tumour with massive pulmonary involvement. BMJ Case Rep. 2011;2011.

17. Liu D, Abolhoda A, Burt ME, Martini N, Bains MS, Downey RJ, et al. Pulmonary metastasectomy for testicular germ cell tumors: A 28year experience. The Annals of Thoracic Surgery.1998;66(5):1709-14.

18. Einhorn LH, Williams SD, Mandelbaum I, Donohue JP. Surgical resection in disseminated testicular cancer following chemotherapeutic cytoreduction. Cancer. 1981;48(4):904-8.

19. Murphy BR, Breeden ES, Donohue JP, Messemer J, Walsh W, Roth BJ, et al. Surgical salvage of chemorefractory germ cell tumors.J Clin Oncol. 1993;11(2):324-9.

20. Wood DP, Herr HW, Motzer RJ, Reuter V, Sogani PC, Morse MJ, et al. Surgical resection of solitary metastases after chemotherapy in patients with nonseminomatous germ cell tumors and elevated serum tumor markers. Cancer. 1992;70(9):2354-7.

21. Giannarini G, Kessler TM, Thoeny HC, Nguyen DP, Meissner C, Studer UE. Do patients benefit from routine follow-up to detect recurrences after radical cystectomy and ileal orthotopic bladder substitution? Eur Urol. 2010;58(4):486-94.

22. Volkmer BG, Kuefer R, Bartsch GC, Jr, Gust K, Hautmann RE. Oncological followup after radical cystectomy for bladder cancer-is there any benefit? JUrol. 2009;181(4):1587-93.

23. von der Maase H, Hansen SW, Roberts JT, Dogliotti L, Oliver T, Moore MJ, et al. Gemcitabine and cisplatin versus methotrexate, vinblastine, doxorubicin, and cisplatin in advanced or metastatic bladder cancer: results of a large, randomized, multinational, multicenter, phase III study. JClin Oncol. 2000;18(17):3068-77.

24. Cowles RS, Johnson DE, McMurtrey MJ. Long-term results following thoracotomy for metastatic bladder cancer. Urology. 1982;20(4):390-2.

25. Siefker-Radtke AO, Walsh GL, Pisters LL, Shen Y, Swanson DA Logothetis CJ, et al. Is there a role for surgery in the management of metastatic urothelial cancer? The M. D. Anderson experience. JUrol. 2004;171(1):145-8.

26. Abe T, Shinohara N, Harabayashi T, Sazawa A, Maruyama S, Suzuki $S$, et al. Impact of multimodal treatment on survival in patients with metastatic urothelial cancer. Eur Urol. 2007:52(4):1106-13.

27. Lehmann J, Suttmann H, Albers P, Volkmer B, Gschwend JE, Fechner G, et al. Surgery for metastatic urothelial carcinoma with curative intent: the German experience (AUO AB 30/05). Eur Urol. 2009;55(6):1293-9.

28. Siefker-Radtke AO, Dinney CP, Shen Y, Williams DL, Kamat AM, Grossman HB, et al. A phase 2 clinical trial of sequential neoadjuvant chemotherapy with ifosfamide, doxorubicin, and gemcitabine followed by cisplatin, gemcitabine, and ifosfamide in locally advanced urothelial cancer: final results. Cancer. 2013;119(3):540-7.

29. Svatek RS, Siefker-Radtke A, Dinney CP. Management of metastatic urothelial cancer: the role of surgery as an adjunct to chemotherapy. Can Urol Assoc J. 2009;3(6 Suppl 4):S228-31.

30. Fabozzi SJ, Schellhammer PF, El-Mahdi AM. Pulmonary metastases from prostate cancer. Cancer. 1995;75(11):2706-9.

31. Saitoh H, Hida M, Shimbo T, Nakamura K, Yamagata J, Satoh T. Metastatic patterns of prostatic cancer: Correlation between sites and number of organs involved. Cancer. 1984;54(12):3078-84.

32. Goto T, Maeshima A, Oyamada Y, Kato R. Solitary pulmonary metastasis from prostate sarcomatoid cancer. World J Surg Oncol. 2010;8:101.

33. Picchio M, Briganti A, Fanti S, Heidenreich A, Krause BJ, Messa $C$, et al. The role of choline positron emission tomography computed tomography in the management of patients with prostate-specific antigen progression after radical treatment of prostate cancer. Eur Urol. 2011;59(1):51-60. 